\title{
Enhancing Vigilance by Low Intensity Transcranial Pulsed Magnetic Stimulation Applying the Entrainment Model
}

\author{
Igor Jerman*, Primož Dovč, Petra Ratajc \\ Physiological Testing Department, BION, Institute for Bioelectromagnetics and New Biology, Ljubljana, Slovenia \\ Email: *igor.jerman@bion.si
}

How to cite this paper: Jerman, I., Dovč, P. and Ratajc, P. (2019) Enhancing Vigilance by Low Intensity Transcranial Pulsed Magnetic Stimulation Applying the Entrainment Model. Open Access Library Journal, 6: e5782.

https://doi.org/10.4236/oalib.1105782

Received: September 12, 2019

Accepted: October 14, 2019

Published: October 17, 2019

Copyright () 2019 by author(s) and Open Access Library Inc.

This work is licensed under the Creative Commons Attribution International License (CC BY 4.0).

http://creativecommons.org/licenses/by/4.0/ (c) (i) Open Access

\begin{abstract}
Vigilance and attention are very important aspects of our working capacities and our general wellbeing as well. Besides taking drugs, they can in principle be achieved also by an appropriate transcranial pulsed magnetic field (MF) stimulation. In the reported research, we took the entrainment principle as the leading one, trying to enhance high beta waves with volunteers. The research conformed to clinical testing conditions. We checked the volunteers' state monitoring their physiological parameters, their responses following visual analog scale (VAS) method and their performance with the Clock test of sustained attention. In addition to observing the differences between the true stimulation and the sham one, we checked also the psychological influence on attention (additional to true stimulation). The results demonstrated overall enhanced relaxation with MF stimulation that was further improved by expectation. Relaxation, better nervous energy conservation and better performance (reduction of errors) may be concluded from all parts of the research.
\end{abstract}

\section{Subject Areas \\ Bioelectromagnetics or Bioengineering}

\section{Keywords}

Magnetic Field Stimulation, Low Intensity rTMS, PEMF, Attention, Relaxation, Conditions of Clinical Trials, Electrophysiological Parameters, Visual Analog Scale (VAS), Clock Test

\section{Introduction}

\subsection{State of the Art}

Vigilance and attention are very important aspects of our working capacities and 
our general wellbeing as well. One possibility to achieve high vigilance is by taking drugs like caffeine, guaranine or cocaine or by having a very good, deep and sufficiently long sleep. However, applying drugs eventually brings undesirable side effects and a need for progressive raising the dose. On the other hand, a constant deep sleep is a rare commodity in our modern stressful way of life. For these reasons, it is very important to look for alternative means to achieve better vigilance and attention, which should be noninvasive, thereby provoking no harmful side effects. One such possibility is an application of a pulsed magnetic stimulation (PEMF) in the region of the head, which can be classified also as a low-intensity rTMS.

Magnetic stimulation of the head region usually utilizes relatively high intensities of magnetic fields of low frequencies and may have multiple healing effects, especially with psychiatric and neurologic disorders [1]. The theoretical background of high-intensity rTMS is based on the magnetic induction of electric fields in the brain region that may excite nervous cells and evoke action potentials or may work inhibitory [2]. Therefore, this kind of MF treatment is invasive to the brain and may bring even harmful side effects like seizures [3]. To turn to another, perhaps a little less effective, but a still promising method of low-intensity rTMS or PEMF stimulation is, therefore, a rational option. Low-intensity magnetic fields cannot elicit nerve cell excitation; neither do they have thermal effects. From the standpoint of mainstream physics, they should not have any measurable biological effects. It is therefore understandable that the mechanism of their effects is still an object of scientific debate and of developing various theoretical models. Some of the latter have also empirical confirmations, yet no one has been universally recognized (see also [4] and [5]). Non-thermic and low induction effects should function on the principle of resonance with some physiological and biochemical processes in the body. If applied to the head, they should influence neuronal excitation patterns and the brainwaves. Since the brainwaves represent a long-range coherent mode of brain cells excitation [6], the applied MF most probably work through resonance. Most probably, they interfere with the endogenous electromagnetic and dipolar oscillations within cells and among them (for a more comprehensive study see [7]).

From the standpoint of general principles of life, the interference with physiological processes may bring two opposing results: 1) enhancing, based on the principle of resonance and 2) inhibitory, because the homeostatic mechanisms of organism's physiology tend to oppose an intrusive external influence. The latter may be similar to radiation hormesis [8], where a small dose of ionizing radiation brings a stimulation of growth, which is in opposition to the effects of the same radiation at higher doses. In connection to this, it is interesting and significant that various authors found both kinds of effects following the MF stimulation of the head region.

The (stimulating) resonance effect is known as entrainment and is frequently expected as a consequence of the MF stimulation of the head region, although 
other stimulation means may be applied, like light and sound [9]. Bell et al. [10] published one of the first reports of the entrainment effect provoked by a sinusoidal MF. They found a non-linear enhancing relation between stimulation and the EEG response, corroborating the entrainment hypothesis. Another, more comprehensive study by Cvetkovic and Cosic [11] comprising four different stimulating frequencies showed that it is possible to influence the alpha and beta waves in stimulated volunteers. However, the effect was not direct under all stimulation conditions and could be even reverse, which corroborates the homeostatic response (inhibition). There are further positive reports regarding the entrainment principle, like the profound study of Thut et al. [12] [13], nevertheless there are also reports that contradict this effect [12] [14] [15]. Cook et al. [16] published a study that tried to find some explanation for these controversies. They found that previous exposure to a pulsed MF sequence determined subjects' responses in the experiment (see also [17]).

When we come to a possibility of influencing vigilance (attention), we may assume an incidental magnetic stimulation will enhance the proportion of brainwaves that are in approximate resonance with the stimulating MF. However, as some researches demonstrate, the entrainment influence may not necessarily comprise only the direct resonant frequency but may entail also its subharmonics and higher harmonics. An interesting study was performed by Ghione and colleagues [18] regarding the effects of 90-min exposure to a homogenous $50 \mathrm{~Hz}$ ELF MF at a flux density of 40 or $80 \mu \mathrm{T}$ applied to the head region. While one would expect an enhancement of high-frequency brainwave bands (gamma, beta), they report a considerable increase in alpha activity after $80 \mu \mathrm{T}$ magnetic treatment compared to the sham exposure. This indicates that here, entrainment followed subharmonics of the stimulating frequency that was approximately two octaves and a half higher than the influenced alpha waves. On the other hand, Gao et al. [19] report an influence that may be ascribed to higher harmonics of the applied PEMF frequency. Here, the influence of ELF PEMF with the frequency of $1 \mathrm{~Hz}$ (intensity: $10 \mathrm{mT}$, stimulation duration: $20 \mathrm{~min}$.) on the human brain was conducted. In comparison with the sham exposure group, the EEG power of theta band $(3.5-7.5 \mathrm{~Hz})$ and lower-alpha band $(7.5-10 \mathrm{~Hz})$ of the stimulation group increased significantly after magnetic stimulation. In direct entrainment, one would expect the stimulation of delta waves $(0-3.5 \mathrm{~Hz})$, yet approximately the second $(4 \mathrm{~Hz})$ and the third $(8 \mathrm{~Hz})$ octaves involving brainwaves were stimulated.

Regarding the MF stimulation of vigilance and attention, not much research has yet been performed. Some researches concentrated on the direct electric current transcranial stimulation and found that it could be a useful fatigue countermeasure and may be more beneficial than caffeine since boosts in performance and mood last longer time [20]. Other authors used sophisticated means to stimulate the spine by using a static magnetic field with a spatial alternation of poles that prevented drowsiness without invoking the REM rebound 
phenomenon [21]. The latter, however, appeared with many other methods of stimulating vigilance and attention. Stimulation of attention was found also with a high intensity extremely low frequency $(1 \mathrm{~Hz})$ rTMS applied to autistic patients [22]. They found a significant error omission rate after rTMS stimulation. On the other side of the intensity spectrum, we can find a report of a case study, in which an applied MF of only $7.5 \mathrm{pT}$ intensity, $4 \mathrm{~Hz}$ frequency, and sinusoidal form significantly increased the mental capacities of a multiple sclerosis patient [23].

\subsection{Background of Present Research, Intentions and Assumptions}

In seeking the best possibility to enhance performance in the sense of higher vigilance and attention, we found that in such psychological states a high frequency in the high beta frequency band increased [24]. In addition to EEG measurements, where we expected the brainwaves would follow the entrainment model, it was our interest to check the influence of MF stimulation also on other physiological states, performance capacities and self-perception of volunteers. For the leading frequency the one close to the border between beta and gamma waves $(25-42 \mathrm{~Hz}$ ) was chosen according to findings published in Lutz et al. [24] and Rubik [25]. The final (exact) determination of the leading frequency followed the so-called GM-scale, found by Meijer and Geesink after a comprehensive study of bioelectromagnetic articles with either stimulatory or inhibitory effects [26]. A supportive frequency of a lesser intensity was chosen as a frequency of water [27] because our organisms are mainly composed of water. This frequency is at the same time in harmonic relationship with the leading frequency (its major sixth) and is close to the gamma/high beta brainwaves. During MF stimulation of volunteers our expectation (research assumption) was as follows:

- brainwaves: enhancing the proportion of beta waves during stimulation, lessening at least one of the three other brainwaves bands (alpha, theta, delta);

- general electrophysiological parameters: calming vegetative nervous system due to rhythmic MF pulsations;

- tendency to sleep: should be lessened due to assumed enhanced vigilance;

- higher accuracy of performance, lower error rate and shorter reaction time with the Clock test of sustained attention

In the research we wanted to check also the influence of expectation in combination with MF stimulation on all three above listed groups of parameters, so in addition to the sham-exposed volunteers (control) and the stimulated ones (verum), we examined parameters of volunteers who a) were stimulated, b) knew that they were stimulated and c) were told what should they expect from the stimulation-the informed situation. Our hypothesis here was that the intentionally provoked expectations in the anticipated direction (higher vigilance) would enhance the influence of MF stimulation.

Although the majority of research in bioelectromagnetics is performed by us- 
ing a homogenous MF, such field is only very rarely applied in practical situations where an MF stimulation may have some practical or therapeutic use. Namely, devices producing highly non-homogenous MFs are mostly offered on the market, which shaped our decision to apply a highly inhomogeneous field to volunteers. The MF was produced by five different coils arranged around the head (see Figure 1). The measures of a coil were as follows $26.5 \mathrm{~mm}$ (length) $\times$ $20 \mathrm{~mm}$ (width) $\times 5.1 \mathrm{~mm}$ (thickness).

\section{Material and Methods}

\subsection{General Data}

\subsubsection{Tested Groups}

The research was performed in May 2019 in the laboratories of the Institute for Bioelectromagnetics and New Biology (BION Institute) in Ljubljana, Slovenia, EU. In the research, 25 volunteers aged from 29 to 76 years (13 men and 12 women) were chosen. The selection criterion was that they had to be disciplined and that they do not suffer from some serious illness, either chronic or acute. They were subject to three different experimental situations:

1) sham stimulation (Control situation);

2) true stimulation (Verum situation);

3) informed true stimulation as explained in the Introduction (Informed situation).

The first two groups were treated in conformity with the clinical trials conditions:

- Prospectiveness (general criteria for the effectiveness of the device's activity were determined in advance);

- Placebo effect ruled out (volunteers did not know whether they were stimulated or not);

- Double blind (neither the volunteer nor the research assistant knew whether MF stimulation was applied or not);

- Randomized (the decisions about sham or true exposure were made randomly).

The volunteers in the Informed situation were intentionally informed about the stimulation; this situation was always applied after the Control and the Verum situations, since the volunteers were the same in all three groups.

The volunteers signed an informed consent in which they agree to cooperate as subjects in this non-invasive scientific research in bioelectromagnetics.

\subsubsection{Stimulating Magnetic Field Characteristics and Positions}

We applied MF stimulation in the region of the head as presented on Figure 1 and described in Table 1. The device generating the MF was manufactured by MDCN Technologies Inc. New York, USA (Omnipemf device) and will be named the device.

During the research, we constantly measured the environmental EM fields and found no significant variation in the frequency range from $5 \mathrm{~Hz}$ to $2 \mathrm{GHz}$ 


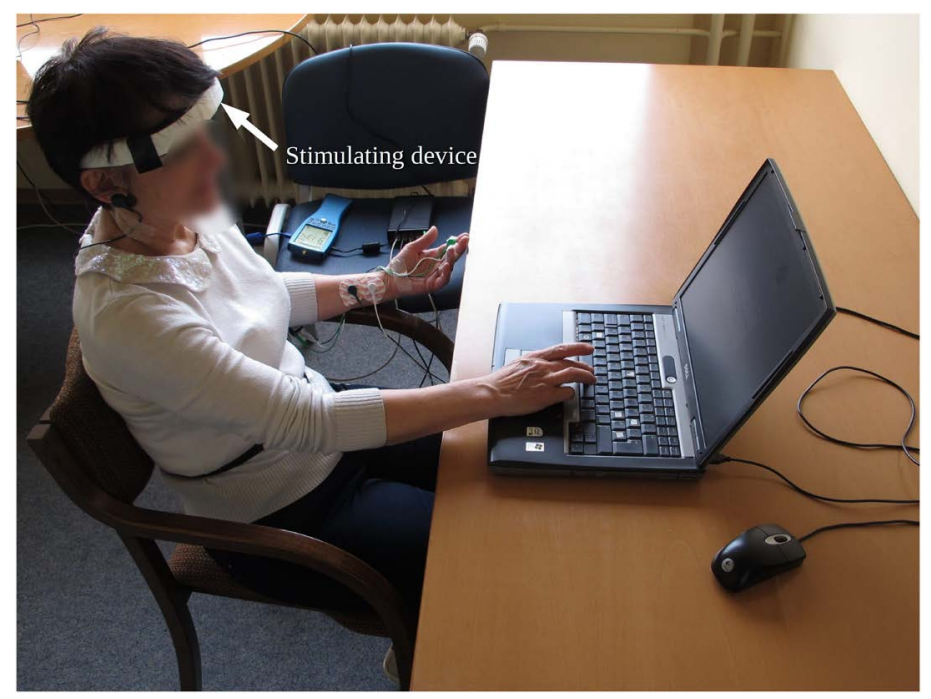

Figure 1. The experimental situation-a volunteer with attached electrodes needed for the measurements of physiological parameters and with the stimulating device in place. Simultaneously she performs the Clock test.

Table 1. Parameters of MF stimulation.

\begin{tabular}{cccc}
\hline $\begin{array}{c}\text { Frequency } \\
(\mathrm{Hz})\end{array}$ & $\begin{array}{c}\text { Magnetic field } \\
\text { intensity (B) }\end{array}$ & Location & Role \\
\hline 33.71 & $2.5 \mathrm{mT}$ & frontal (1 coil) & $\begin{array}{c}\text { resonant stimulative } \\
\text { frequency, found in literature }\end{array}$ \\
54 & $0.25 \mathrm{mT}$ & $\begin{array}{c}\text { frontal }(1 \text { coil), temporal lobes left } \\
(2 \text { coils }) \text { and right }(2 \text { coils })\end{array}$ & supportive stimulation \\
\hline
\end{tabular}

between different days. The geomagnetic field varied from 47,963 to 48,002 $\mathrm{nT}$ (data from the nearest INTERMAGNET [28] observatory located in Lonjsko polje, Croatia).

\subsection{Physiological Testing and Exposure Procedure}

Each volunteer attended the measurements thrice at the same hour of the day. At the arrival, he/she filled out the questionnaire and completed the visual analog scale (VAS) test (see 2.4. for more detail). After that research assistant placed the device on his/her head in an appropriate position (Figure 1) and attached the electrodes needed for the measurements of physiological parameters. All the preparations before the measurements took about 10 minutes and during this time the device was already stimulating volunteers. This 10 minutes pre-treatment was intended to extend the stimulation period to about 40 minutes in total. During the measurements, which lasted for 30 minutes, volunteers sat in a wooden chair in front of a computer and were doing the modified Clock test from the PEBL Psychological Test Battery [29]. This test is a computerized version of the Mackworth Clock device [30] and is used in the field of experimental psychology to study the effects of long-term vigilance on the detection of signals. The test took approximately 30 minutes to complete. During this time, volunteers had to 
react with the space key on a keyboard when dark dot skipped one position on a circular orbit. For the test to be as monotone and tedious as possible, skips happened only in approximately $0.8 \%$ of total dot shifts. The following parameters were measured during this time: brain wave activity (EEG) in four standard frequency spectra (beta waves from 14 to $30 \mathrm{~Hz}$, alpha waves from 8 to $14 \mathrm{~Hz}$, theta waves from 4 to $8 \mathrm{~Hz}$ and delta waves from 0 to $4 \mathrm{~Hz}$ ), skin conductance, heart rate, respiration rate, finger temperature, heart rate variability and thorax expansion depth. The positive electrode for the EEG measurement was placed on the F3 position of the international 10 - 20 EEG system [31], while the negative and ground electrodes were placed on the right and the left earlobe, respectively. After the removal of the electrodes, the volunteer again completed the VAS test and the questionnaire enabling the comparison between before and after for each tested situation.

We calculated thirty-second medians for every volunteer. On the basis of these data, we calculated aggregated thirty-second medians for all 25 volunteers and used this data to draw graphs for each measured parameter and for further statistical analysis. For the sake of analysis and interpretation of the results, 30 minutes of measurements were split into three parts (each consisting of 20 data points with calculated 30 -second median):

- first part-from 0 to 10 minutes,

- second part-from 10 to 20 minutes,

- third part-from 20 to 30 minutes.

For calculating statistically significant differences between any of the three experimental situations we used the Friedman test. With a post-hoc test (Wilcoxon Signed-Rank test) we determined which comparison demonstrated these differences. The outcome of the results for all tests was corrected by the Holm-Bonferroni correction for multiple comparisons [32].

\subsection{Clock Test of Sustained Attention}

A total of 4500 dot shifts occurred in a Clock test in 30 minutes out of which there were approximately 36 dot skips $(0.8 \%)$. Because the number of skips randomly varied between volunteers, we calculated the proportion of correctly detected skips out of all skips (CSoS). A number of errors were recorded if a volunteer reacted when there was no skip, from which the proportion of error responses out of total dot shifts was calculated (FRoA). Additionally, correct responses out of all dot shifts were calculated (CRoA). Two kinds of responses were evaluated as correct: 1) response when there was a skip and 2) no response when there was no skip (only shift), see Figure 2 for better understanding. Reaction time was recorded between the moment a skip happened and the moment a volunteer reacted to it (RT).

We used the Friedman test to figure out whether there were any statistically significant differences. Additionally, we conducted post hoc tests (Wilcoxon Signed-Rank Test) to determine exactly between which two situations significant differences demonstrated themselves. 


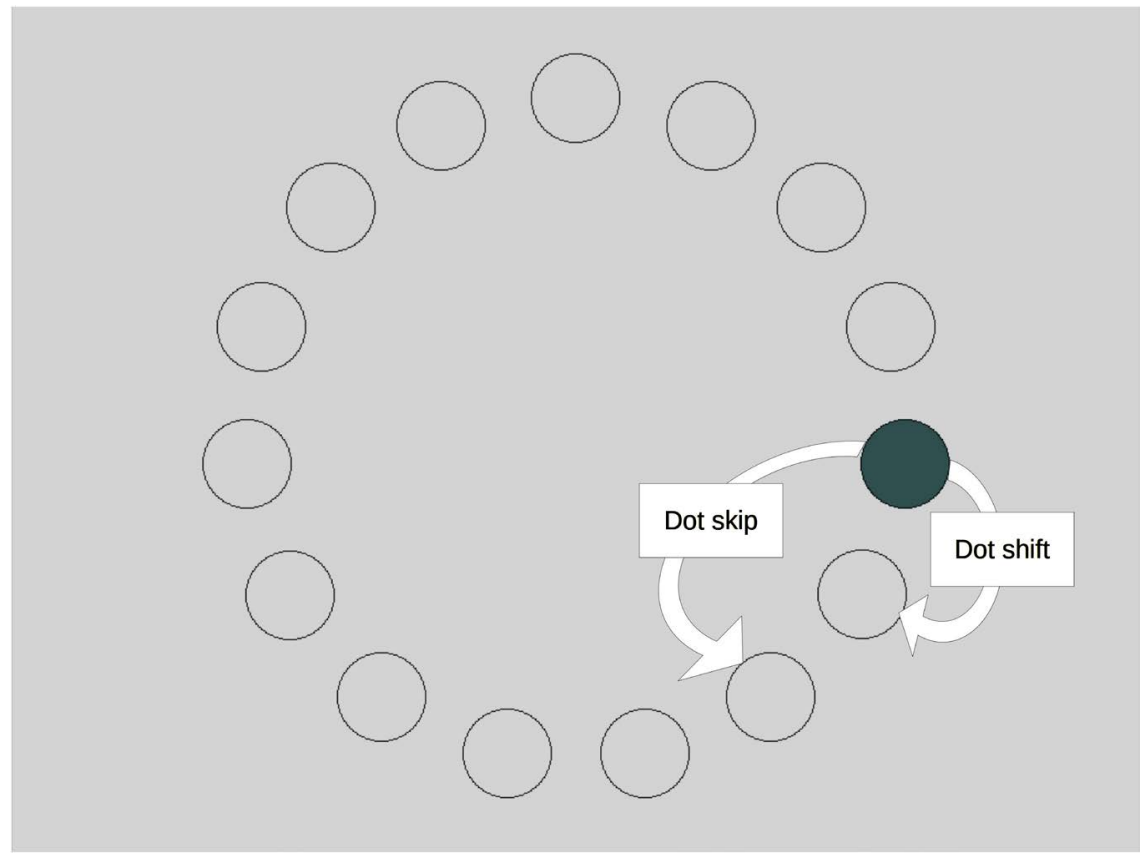

Figure 2. A screenshot of the Clock test of sustained attention. An explanation of a dot skip and a dot shift is labeled for a better notion of the test.

\subsection{The Visual Analogue Scale (VAS)}

The Visual Analogue Scale (VAS) has been in use for the measurement of subjective parameters that are impossible to measure by any physical method, like pain, anxiety, quality of sleep, etc. since the 1920s [33]. Today it can be used independently or as a supplement to other measurements and may be used even in pharmacology [34]. The scale consists of a line that is usually $100 \mathrm{~mm}$ in length. On each side, there is an anchor descriptor such as very unrelaxed-very relaxed. The volunteer makes a mark on this (normally and in our case horizontal) line, reflecting his/her perception. Afterward, the distance from the left endpoint to the mark is measured in mm. In our case, the VAS line was displayed on the computer screen and the volunteer had to mark the position between the descriptors by using a mouse and clicking the determined point. VAS measurements enable a consequent statistical analysis of data as in any technical device-based measurement systems.

We used VAS scale for evaluation of the subjective perception of three different parameters: relaxation, concentration and energy level. VAS scale was included in online survey volunteers completed before and after each measurement. The online open-source application for web surveys [35] determined the marked position as a distance from the left point in millimeters (range 0 - 100), which was considered as the VAS score. We determined mean VAS scores obtained before and after each measurement, and compared them using t-test. We calculated a change of VAS scores for each individual person (after-before the individual measurement), and used paired t-test for the comparisons of different situations (Control, Verum, Informed). 
Measurements were carried out in a double-blind and randomized fashion, following the same conditions for clinical testing as described in section 2.1.1 (Table 2).

Besides VAS testing, a question as to when a volunteer expected to be under the true stimulation as well as when they felt more focused was asked after the second testing.

\section{Results}

\subsection{Physiological Testing}

Results concerning electrophysiological measurements demonstrate statistically significant differences between three experimental situations for the following parameters: skin conductance, heart rate, finger temperature, the proportion of alpha and delta waves and thorax expansion depth (Table 3, Wilcoxon signedrank test). Skin conductance, heart rate, and finger temperature demonstrated differences in all three parts of measurements.

SC was substantially lower for both the Verum and the Informed situations

Table 2. Questions and anchor descriptors for evaluation of three different parameters.

\begin{tabular}{ccc}
\hline Parameter & Question & Anchor descriptors \\
\hline Relaxation & How relaxed do you feel at the moment? & Very unrelaxed-very relaxed \\
Concentration & How focused do you feel at the moment? & Very unfocused-very focused \\
Energy level & How full of energy do you feel at the & Complete lack of energy-full of \\
& & energy
\end{tabular}

Table 3. p-values of the Friedman test based on 30-second medians for each parameter during the three parts of measurements. Holm-Bonferroni correction for multiple comparisons is applied to the p-values in the table. Bold values denote statistically significant differences between at least two of the three different experimental situations $(\mathrm{p}<0.05)$, values with regular black font represent statistically marginally significant differences between at least two of the three different experimental situations ( $\mathrm{p}<0.1$ ). Marks: $\mathrm{SC}$ - skin conductance, $\mathrm{RR}$ - respiration rate, $\mathrm{HR}$ - heart rate, TMP - finger temperature, beta, alpha, theta, and delta-the proportion of beta, alpha, theta and delta spectrum of brain activity, HRV—-heart rate variability, TED—-thorax expansion depth.

\begin{tabular}{cccc}
\hline & $0-10 \mathrm{~min}$ & $10-20 \mathrm{~min}$ & $20-30 \mathrm{~min}$ \\
\hline SC & 0.000 & 0.000 & 0.000 \\
RR & 0.578 & 0.159 & 0.521 \\
HR & 0.004 & 0.011 & 0.002 \\
TMP & 0.000 & 0.000 & 0.000 \\
beta & 1.000 & 0.668 & 0.668 \\
alfa & 0.003 & 0.668 & 0.668 \\
theta & 0.011 & 0.210 & 0.210 \\
delta & 0.002 & 0.007 & 0.007 \\
HRV & 1.000 & 0.668 & 0.668 \\
TED & 0.000 & 0.268 & 1.000 \\
\hline
\end{tabular}


during the entire measurements when compared to the Control one (Figure 3). Lower SC indicates deeper relaxation of volunteers [36]. However, SC indicates that expectation additionally lowered its values in the Informed situation when compared to the Verum one (a significant difference in the first part of measurements). There were also substantial differences in initial values for this parameter because volunteers have already been stimulated for 10 minutes before the start of measurements (Table 4).

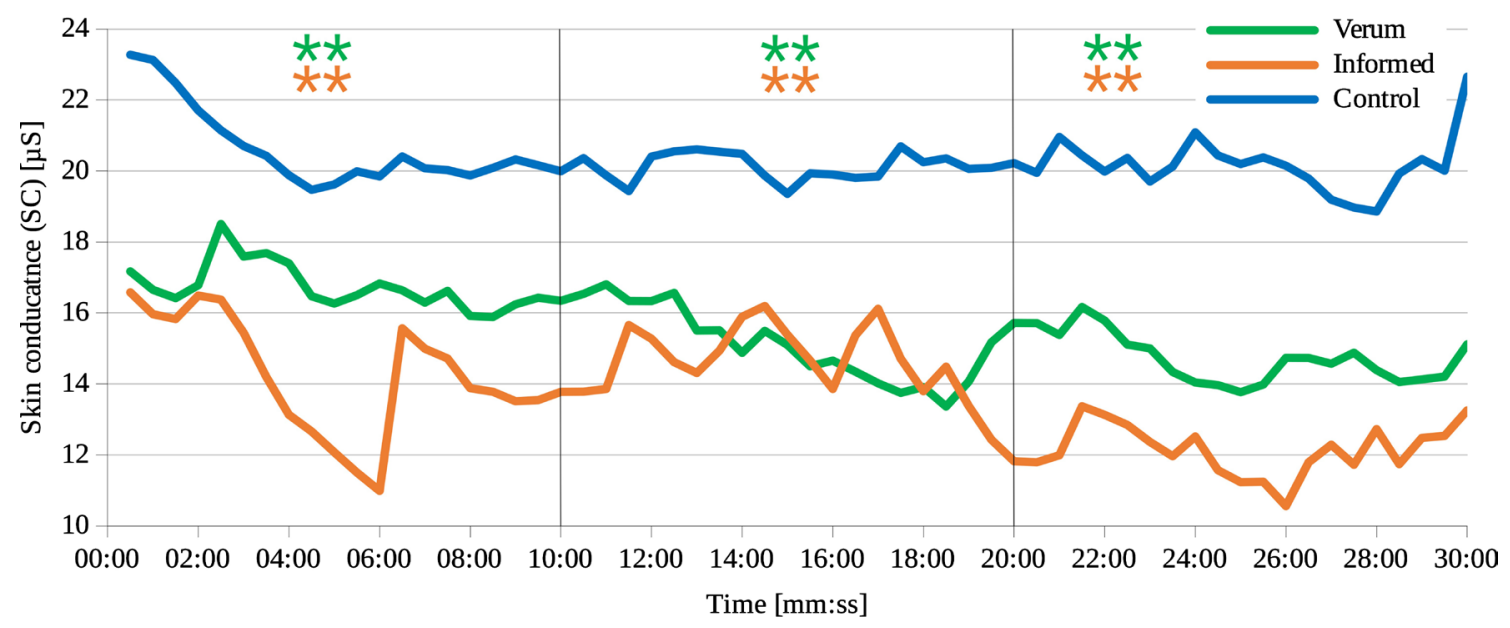

Figure 3. Skin conductance (SC) from twenty-five volunteers (Verum, Informed and Control situations). Asterisks denote statistically significant differences $\left({ }^{*} p<0.01\right)$ in three parts of measurements separated by vertical grey lines. Green asterisks: differences between Verum and Control, orange asterisks: differences between Informed and Control. Statistical differences for comparison between Verum and Informed situations are not shown.

Table 4. p-values of the post-hoc test (Wilcoxon Signed-Rank Test) based on 30-second medians for each parameter during the three parts of measurements. Holm-Bonferroni correction for multiple comparisons is applied to the p-values in the table. Values with bold black font represent statistically significant differences between two of the selected situations ( $p<0.05)$, values with regular black font represent statistically marginally significant differences between two of the selected situations $(\mathrm{p}<0.1)$. Marks: SC-skin conductance, RR-respiration rate, HR-heart rate, TMP-finger temperature, beta, alpha, theta and delta-the proportion of beta, alpha, theta, and delta spectrum of brain activity, HRV—heart rate variability, TED—thorax expansion depth.

\begin{tabular}{ccccccccccc}
\hline \multirow{2}{*}{$\begin{array}{c}\text { Comparison } \\
\text { pair }\end{array}$} & $\begin{array}{c}\text { Verum } \\
\text { Control }\end{array}$ & $\begin{array}{c}\text { Informed } \\
\text { Control }\end{array}$ & $\begin{array}{c}\text { Verum } \\
\text { Informed }\end{array}$ & $\begin{array}{c}\text { Verum } \\
\text { Control }\end{array}$ & $\begin{array}{c}\text { Informed } \\
\text { Control }\end{array}$ & $\begin{array}{c}\text { Verum } \\
\text { Informed }\end{array}$ & $\begin{array}{c}\text { Verum } \\
\text { Control }\end{array}$ & $\begin{array}{c}\text { Informed } \\
\text { Control }\end{array}$ & $\begin{array}{c}\text { Verum } \\
\text { Informed }\end{array}$ \\
\hline SC & $\mathbf{0 . 0 0 8}$ & $\mathbf{0 . 0 0 8}$ & $\mathbf{0 . 0 0 8}$ & $\mathbf{0 . 0 0 8}$ & $\mathbf{0 . 0 0 8}$ & 1.000 & $\mathbf{0 . 0 0 8}$ & $\mathbf{0 . 0 0 8}$ & $\mathbf{0 . 0 0 8}$ \\
RR & 1.000 & 1.000 & 1.000 & 1.000 & 1.000 & 0.060 & 1.000 & 1.000 & 1.000 \\
HR & 0.278 & $\mathbf{0 . 0 1 6}$ & 0.373 & 0.054 & $\mathbf{0 . 0 2 4}$ & 1.000 & $\mathbf{0 . 0 1 1}$ & 0.197 & 0.908 \\
TMP & 0.178 & $\mathbf{0 . 0 0 8}$ & 0.373 & $\mathbf{0 . 0 0 8}$ & $\mathbf{0 . 0 0 8}$ & 1.000 & 0.111 & $\mathbf{0 . 0 0 8}$ & $\mathbf{0 . 0 0 8}$ \\
beta & 1.000 & 1.000 & 1.000 & 1.000 & 0.646 & 1.000 & 1.000 & 1.000 & 1.000 \\
alpha & 0.087 & 0.054 & 1.000 & 0.606 & 0.373 & 1.000 & 0.019 & 0.840 & 1.000 \\
theta & 0.278 & 1.000 & 0.124 & 1.000 & 1.000 & 1.000 & 0.530 & 0.606 & 1.000 \\
delta & 1.000 & $\mathbf{0 . 0 4 2}$ & 0.278 & 0.395 & $\mathbf{0 . 0 2 4}$ & 0.701 & 1.000 & 1.000 & 0.530 \\
HRV & 1.000 & 1.000 & 1.000 & 1.000 & 1.000 & 1.000 & 1.000 & 1.000 & 1.000 \\
TED & $\mathbf{0 . 0 0 8}$ & $\mathbf{0 . 0 1 1}$ & 0.530 & 0.395 & 0.556 & 1.000 & 1.000 & 1.000 & 1.000 \\
\hline
\end{tabular}


TED demonstrated significant differences only in the first part of the measurements where values for the Control situation were higher as compared to both the Verum and the Informed situations (Figure 4). After 13 minutes this parameter suddenly dropped for the Control situation, after which there were no differences between the three situations. Lower TED is correlated with a lower concentration of adrenaline (within physiological concentrations; see [37]), which is one of the stress hormones and stimulator of the sympathetic nervous system. This result is therefore in harmony with SC results.

HR was lower for both the Verum and the Informed situations in comparison to the Control one at least during one part of the measurements (Figure 5). The

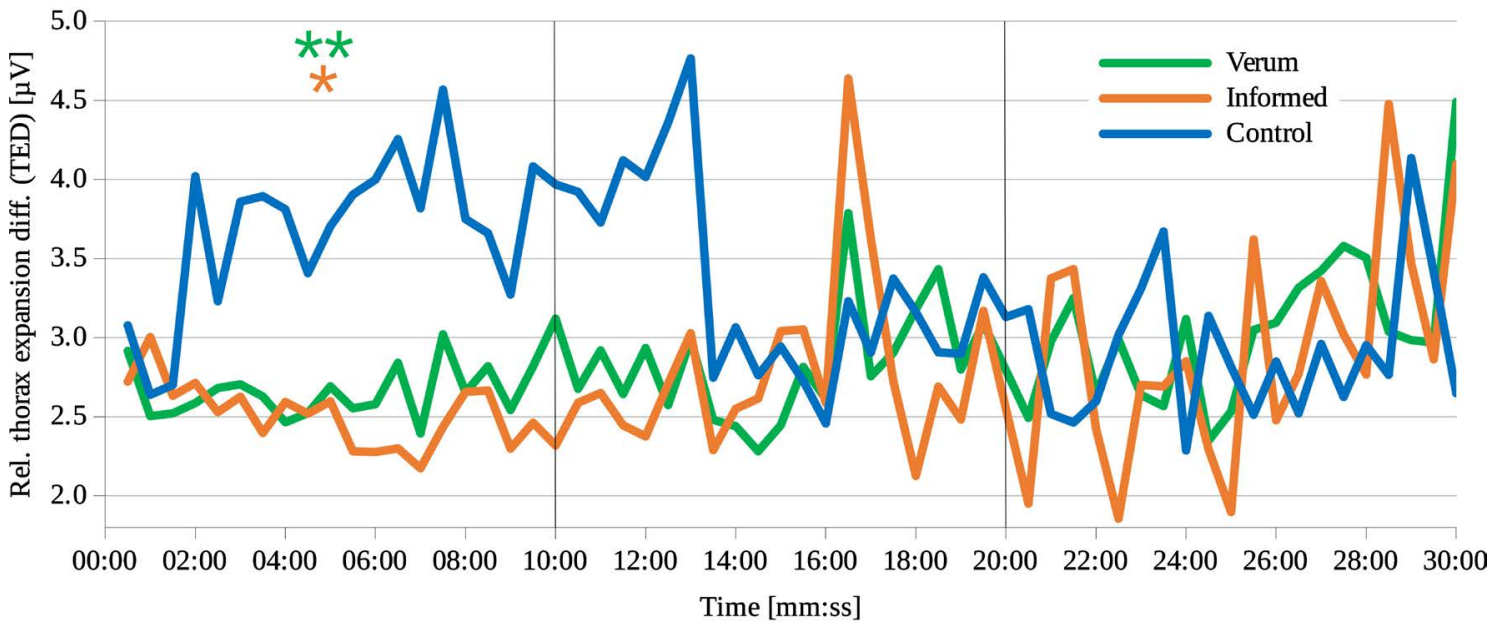

Figure 4. Relative thorax expansion difference (TED) from twenty-five volunteers (Verum, Informed and Control situations). Asterisks denote statistically significant differences $\left({ }^{*} p<0.05 ;{ }^{* *} p<0.01\right)$ in three parts of measurements separated by vertical grey lines. Green asterisks: differences between Verum and Control, orange asterisks: differences between Informed and Control. Statistical differences for comparison between Verum and Informed situations are not shown.

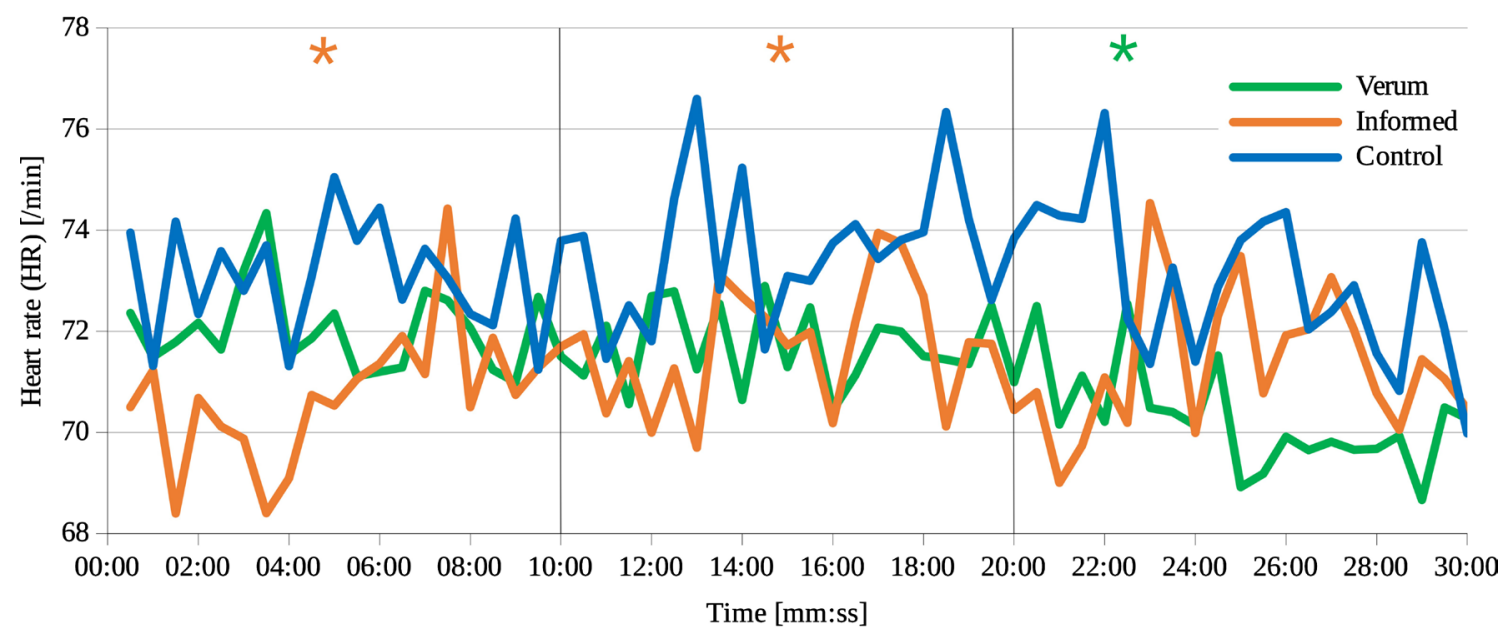

Figure 5. Heart rate (HR) from twenty-five volunteers (Verum, Informed and Control situations). Asterisks denote statistically significant differences $\left({ }^{\star} \mathrm{p}<0.05\right)$ in three parts of measurements separated by vertical grey lines. Green asterisks: differences between Verum and Control, orange asterisks: differences between Informed and Control. Statistical differences for comparison between Verum and Informed situations are not shown. 
Verum situation demonstrated significant differences in the third part of the measurements, while the Informed one demonstrated them in the first two parts. In general, both the Verum and the Informed situations showed a lower HR than the Control one which indicates a greater calmness and relaxation and which is again in accordance with both SC and TED.

TMP was significantly higher in both the Verum and the Informed situations when compared to the Control one; the Informed situation yielded even slightly higher values than the Verum one (Figure 6). Expectations for the Informed situation intensified the device's influence on TMP. A lower TMP was found to correlate with stress exposure [38]. Therefore, we may safely assume that a higher peripheral temperature corresponds to a more relaxed state.

The proportion of alpha waves was statistically significantly higher only in the last part of measurements for the Verum situation when compared to the Control one (Figure 7). Higher alpha waves for the Verum situation indicate a slightly increased relaxation. Even if there is only a slight difference in alpha waves between the three experimental situations there is a prominent difference in linear trends. It is slightly positive for both the Verum and the Informed situations while it is negative for the Control situation. This speaks about increasing relaxation for both MF stimulated situations.

The proportion of delta waves was significantly higher in the first two parts of measurements in the Informed situation when compared to the Control one (Figure 8). The linear trend for both the Verum and the Informed situations was slightly negative while it was slightly positive for the Control situation. We ascribe the difference in the first part to a higher overall relaxation as shown by many other parameters, while the negative trend may be a consequence of the expected (gradual) entrainment, where there should be a relative diminution of low-frequency brain waves. There was no difference in beta waves, as seen on Figure 9.

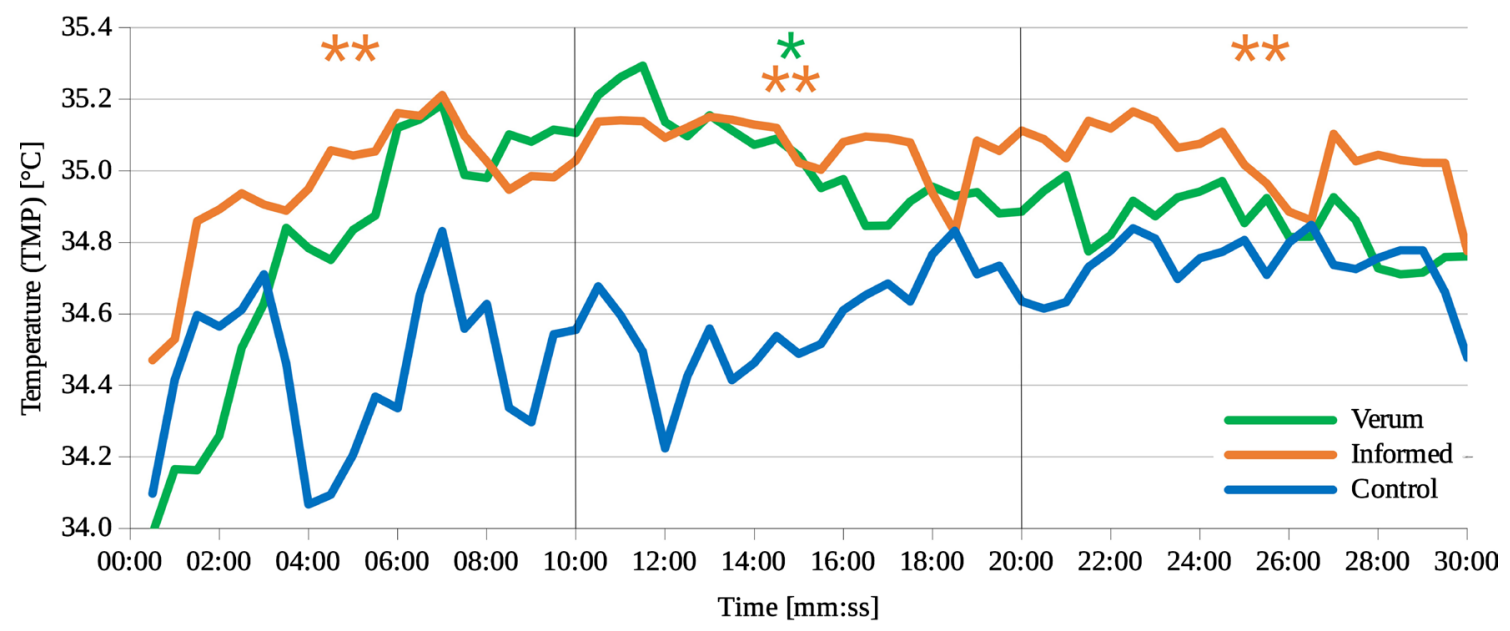

Figure 6. Finger temperature (TMP) from twenty-five volunteers (Verum, Informed and Control situations). Asterisks denote statistically significant differences $\left({ }^{*} \mathrm{p}<0.05 ;{ }^{*} \mathrm{p}<0.01\right)$ in three parts of measurements separated by vertical grey lines. Green asterisks: differences between Verum and Control, orange asterisks: differences between Informed and Control. Statistical differences for comparison between Verum and Informed situations are not shown. 


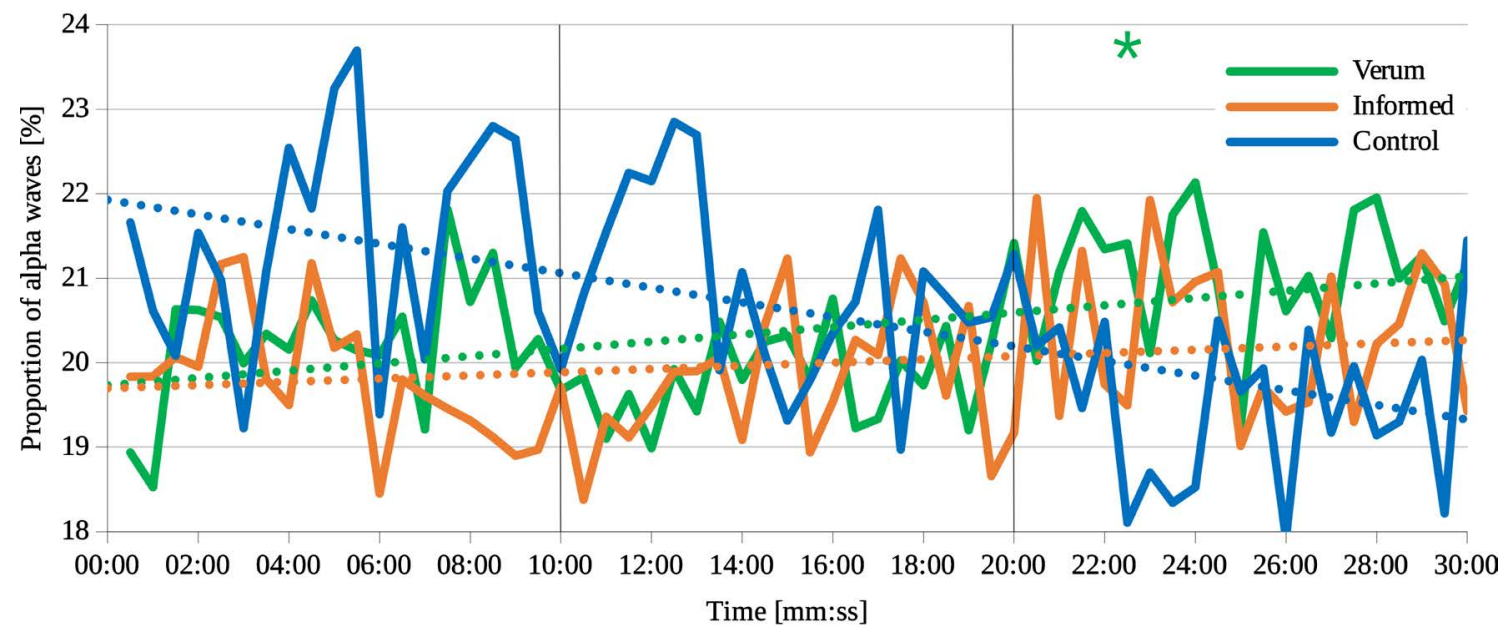

Figure 7. Proportion of alpha waves from twenty-five volunteers (Verum, Informed and Control situations). Asterisks denote statistically significant differences $\left({ }^{*} p<0.05\right)$ in three parts of measurements separated by vertical grey lines. Green asterisk: the difference between Verum and Control. Statistical differences for comparison between Verum and Informed situations are not shown.

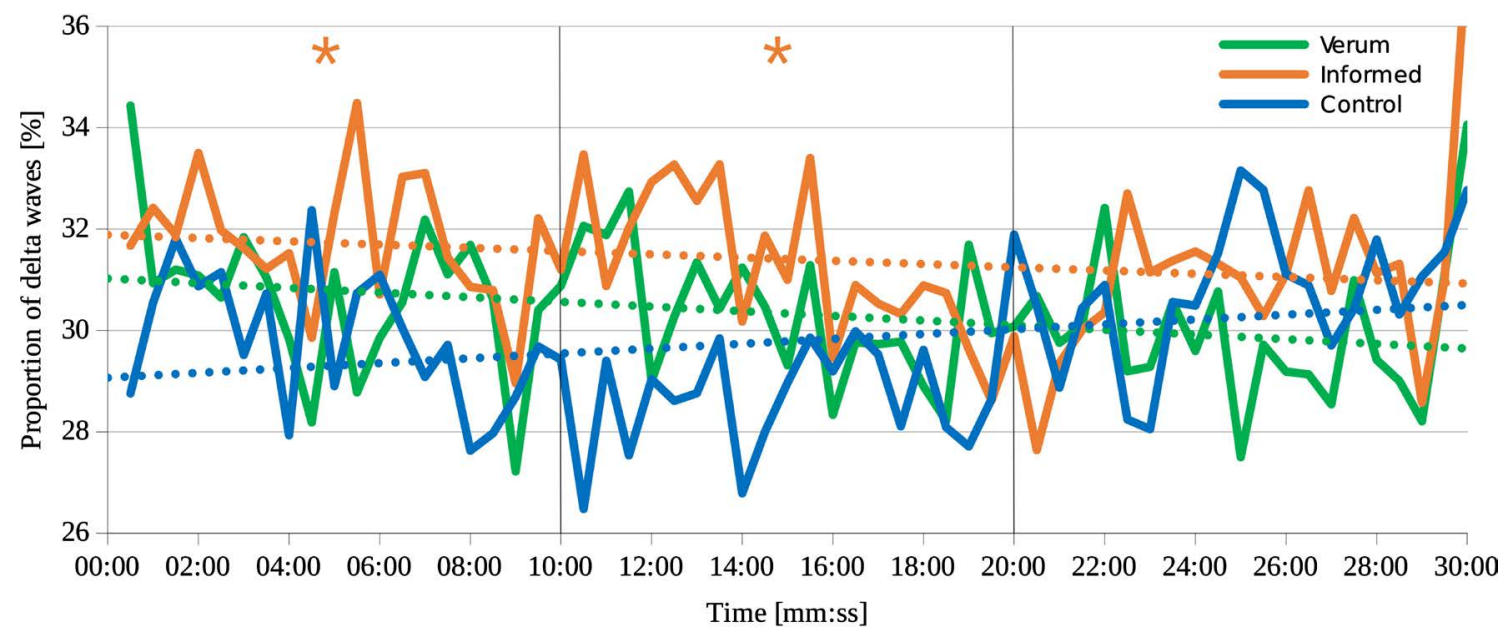

Figure 8. Proportion of delta waves from twenty-five volunteers (Verum, Informed and Control situations). Asterisks denote statistically significant differences $\left({ }^{*} p<0.05\right)$ in three parts of measurements separated by vertical grey lines. Orange asterisks: differences between Informed and Control. Statistical differences for comparison between Verum and Informed situations are not shown.

Mean values with standard error of measured parameters for all three situations and for all three parts of measurement are presented in Table 5.

\subsection{Clock Test of Sustained Attention}

Statistical analysis demonstrated a significant difference between the Informed and the Control situation for the proportion of error responses out of total dot shifts (FRoA, Table 7). Although the Friedman test demonstrated significant differences for mean reaction time too (Table 6), there was no difference in the post-hoc test for the comparison of three situations (Table 7), nevertheless, from the results, we may assume that the Informed situation enhanced this parameter vs. the other two ones. 


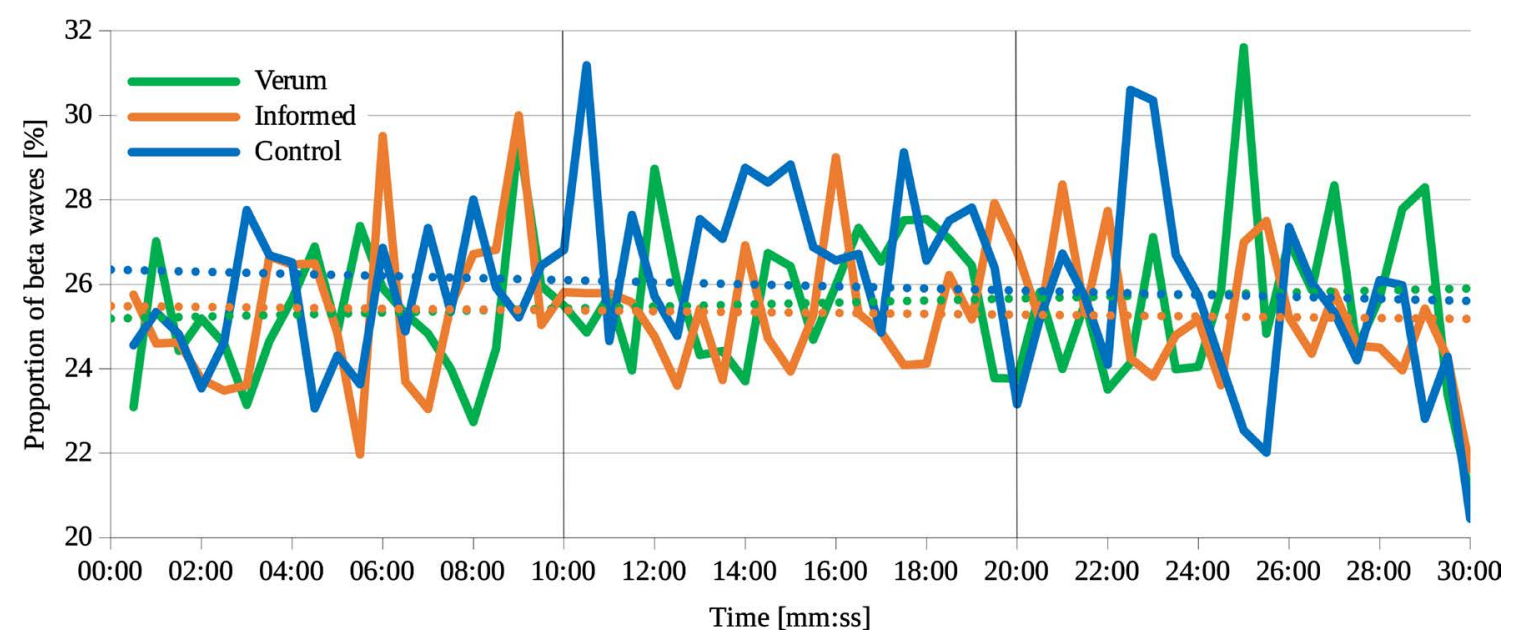

Figure 9. Proportion of beta waves from twenty-five volunteers (Verum, Informed and Control situations).

Table 5. Overview of the mean values for all measured parameters with \pm standard error $(\mathrm{N}=20)$ for all three test situations. Marks: SC—skin conductance, RR — respiration rate, HR - heart rate, TMP—finger temperature, beta, alpha, theta, and delta-the proportion of beta, alpha, theta and delta spectrum of brain activity, HRV—-heart rate variability, TED—thorax expansion depth.

\begin{tabular}{|c|c|c|c|c|c|c|c|c|c|}
\hline & & $0-10 \min$ & & & $10-20 \mathrm{~min}$ & & & $20-30 \mathrm{~min}$ & \\
\hline & Verum & Informed & Control & Verum & Informed & Control & Verum & Informed & Control \\
\hline SC & $16.732 \pm 0.145$ & $14.251 \pm 0.372$ & $20.63 \pm 0.217$ & $15.129 \pm 0.233$ & $14.528 \pm 0.26$ & $20.131 \pm 0.086$ & $14.704 \pm 0.153$ & $12.157 \pm 0.163$ & $20.175 \pm 0.186$ \\
\hline $\mathrm{RR}$ & $17.688 \pm 0.108$ & $17.239 \pm 0.121$ & $17.552 \pm 0.155$ & $17.098 \pm 0.094$ & $16.511 \pm 0.172$ & $17.183 \pm 0.121$ & $16.615 \pm 0.121$ & $16.176 \pm 0.175$ & $16.704 \pm 0.173$ \\
\hline HR & $72.012 \pm 0.186$ & $70.778 \pm 0.296$ & $73.13 \pm 0.248$ & $71.691 \pm 0.176$ & $71.668 \pm 0.277$ & $73.639 \pm 0.317$ & $70.263 \pm 0.228$ & $71.39 \pm 0.314$ & $72.916 \pm 0.342$ \\
\hline TMP & $34.781 \pm 0.084$ & $34.959 \pm 0.041$ & $34.447 \pm 0.046$ & $35.03 \pm 0.031$ & $35.078 \pm 0.018$ & $34.583 \pm 0.032$ & $34.851 \pm 0.02$ & $35.035 \pm 0.022$ & $34.735 \pm 0.019$ \\
\hline beta & $25.607 \pm 0.326$ & $25.644 \pm 0.324$ & $25.585 \pm 0.324$ & $26.797 \pm 0.344$ & $26.74 \pm 0.343$ & $27.01 \pm 0.357$ & $25.396 \pm 0.587$ & $25.314 \pm 0.565$ & $25.316 \pm 0.57$ \\
\hline alpha & $21.267 \pm 0.306$ & $21.306 \pm 0.303$ & $21.397 \pm 0.295$ & $20.694 \pm 0.271$ & $20.804 \pm 0.275$ & $20.925 \pm 0.245$ & $19.53 \pm 0.25$ & $19.583 \pm 0.25$ & $19.495 \pm 0.219$ \\
\hline delta & $30.073 \pm 0.321$ & $29.98 \pm 0.31$ & $29.834 \pm 0.297$ & $29.226 \pm 0.344$ & $29.152 \pm 0.345$ & $28.802 \pm 0.254$ & $30.639 \pm 0.346$ & $30.614 \pm 0.339$ & $30.756 \pm 0.313$ \\
\hline HRV & $10.808 \pm 0.436$ & $10.844 \pm 0.348$ & $11.57 \pm 0.56$ & $12.889 \pm 0.373$ & $12.42 \pm 0.372$ & $11.826 \pm 0.4$ & $12.594 \pm 0.68$ & $12.075 \pm 0.499$ & $13.431 \pm 0.488$ \\
\hline TED & $2.678 \pm 0.043$ & $2.51 \pm 0.045$ & $3.681 \pm 0.108$ & $2.828 \pm 0.08$ & $2.797 \pm 0.124$ & $3.33 \pm 0.139$ & $3.05 \pm 0.109$ & $2.92 \pm 0.157$ & $2.919 \pm 0.104$ \\
\hline
\end{tabular}

Table 6. p-values of Friedman based on averages of 25 volunteers for different parameters. Holm-Bonferroni correction for multiple comparisons is applied to the p-values in the table. Bold values denote statistically significant differences between at least two of the three different experimental situations ( $\mathrm{p}<0.05$ ). Marks: CSoS—a proportion of correctly detected skips out of total skips, FRoA-a proportion of error responses out of total shifts, CRoA-a proportion of correctly detected skips out of total shifts, $\mathrm{RT}-$ mean reaction time.

\begin{tabular}{cc}
\hline Parameter & p-value \\
\hline CSoS & 0.782 \\
FRoA & 0.013 \\
CRoA & 0.782 \\
RT & 0.034 \\
\hline
\end{tabular}


The proportion of error halved from around $0.13 \%$ in the Control situation to around $0.06 \%$ in the Informed one (Figure 10 ). This parafmeter decreased to about $0.08 \%$ in the Verum situation, which is very close to the Informed one but still not enough for the statistically significant difference when compared to the Control one.

Although the reaction time did not demonstrate a statistically significant difference between different experimental situations the average for the Informed situation was still much lower than for the other two situations (around $570 \mathrm{~ms}$ while it was around $610 \mathrm{~ms}$ for the Control and the Verum situations; Figure 11).

Mean values with standard error for all measured parameters and for all three situations are presented in Table 8.

\subsection{Visual Analog Scale (VAS)}

After surveying all VAS measurements we estimated that only the scores from 21

Table 7. p-values of the post-hoc test (Wilcoxon signed-rank test) based on averages of 25 volunteers for different parameters. Holm-Bonferroni correction for multiple comparisons is applied to the p-values in the table. Bold values denote statistically significant differences between at least two of the three different experimental situations $(p<0.05)$. Marks: CSoS-proportion of correctly detected skips of total skips, FRoA-proportion of error responses of total dot shifts, CRoA-proportion of correctly detected skips of total shifts, RT-mean reaction time.

\begin{tabular}{cccc}
\hline Comparison pair & Verum Control & Informed Control & Verum Informed \\
\hline CSoS & 1.000 & 1.000 & 1.000 \\
FRoA & 1.000 & 0.016 & 1.000 \\
CRoA & 1.000 & 1.000 & 1.000 \\
RT & 1.000 & 0.131 & 0.230 \\
\hline
\end{tabular}

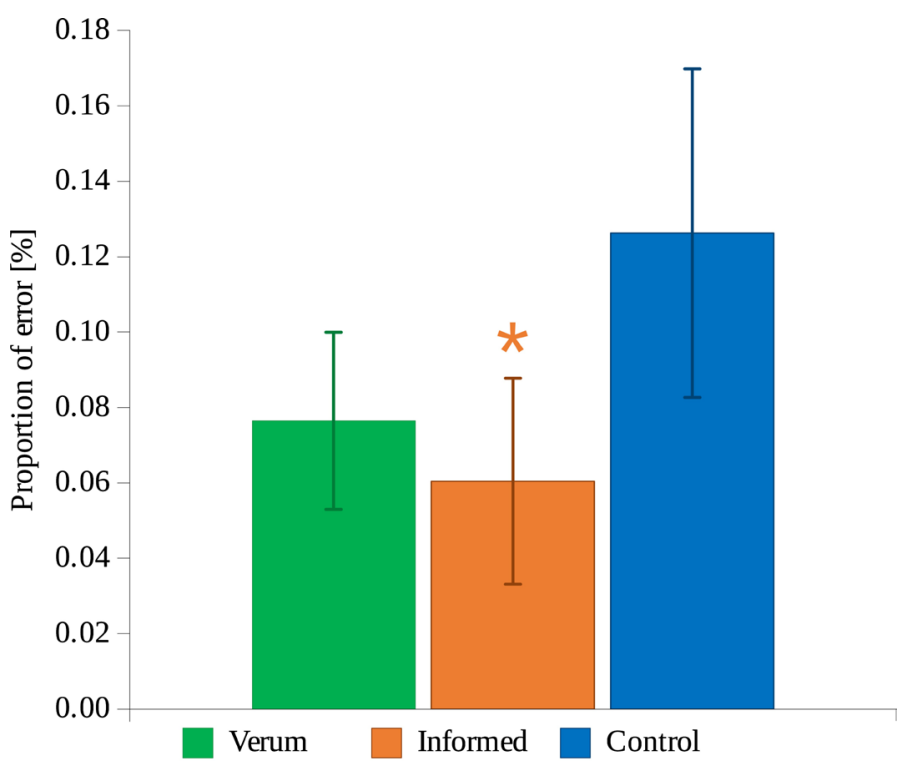

Figure 10. Proportion of error responses of total dot shifts $\pm S E(N=25)$ in Clock test for the Verum, Informed and the Control situations. The asterisk denote statistically the significant difference $\left({ }^{*} \mathrm{p}<0.05\right)$ between Informed and Control. 


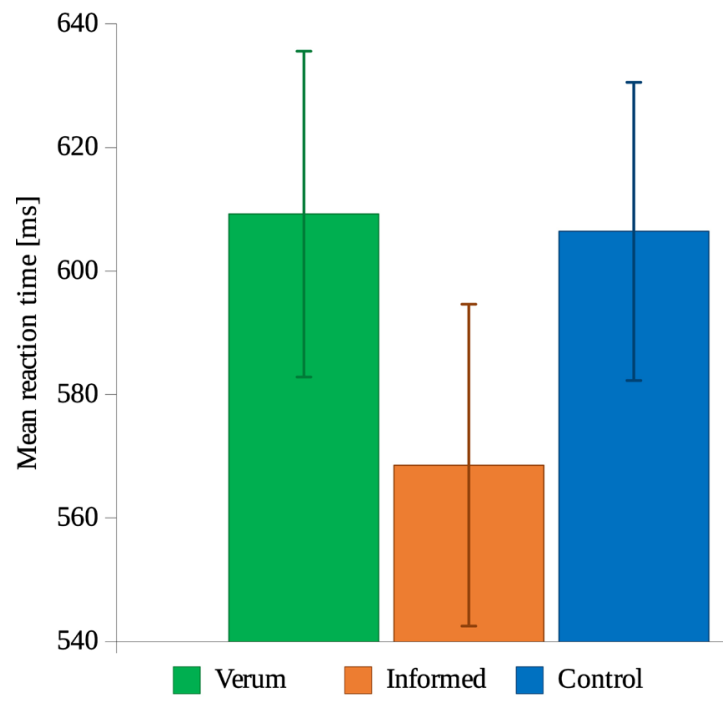

Figure 11. Mean reaction time $\pm \mathrm{SE}(\mathrm{N}=25)$ in Clock test for the Verum, Informed and the Control situations.

Table 8. Overview of the mean values for all measured parameters with \pm standard error $(\mathrm{N}=20)$ for all three test situations. Marks: CSoS-proportion of correctly detected skips of total skips, FRoA-proportion of error responses of total dot shifts, CRoA-proportion of correctly detected skips of total shifts, RT-mean reaction time.

\begin{tabular}{ccccc}
\hline & CSoS [\%] & FRoA [\%] & CRoA [\%] & RT [ms] \\
\hline Verum & $81.337 \pm 3.263$ & $0.076 \pm 0.023$ & $99.759 \pm 0.044$ & $609.22 \pm 26.376$ \\
Informed & $81.997 \pm 3.807$ & $0.06 \pm 0.027$ & $99.774 \pm 0.048$ & $568.575 \pm 26.056$ \\
Control & $83.228 \pm 2.909$ & $0.126 \pm 0.044$ & $99.739 \pm 0.053$ & $606.413 \pm 24.14$ \\
\hline
\end{tabular}

volunteers were suitable for analysis. Results from four volunteers were excluded due to high initial VAS scores, which did not allow a reliable evaluation of changes during the treatments. The VAS scores showed higher relaxation, lower focus and lower energy level after treatment compared to the baseline (Figure 12). Volunteers felt the most relaxed after the Verum situation. However, changes in VAS scores before and after the measurements were not statistically significant, and there were also no significant differences between situations (see Table 9).

After completed Control and Verum situations, volunteers were asked to evaluate which measurements made them feel more focused. Due to double-blind and randomized research setting, the possible answers were (A) today's measurement made me feel more focused, (B) the previous measurement made me feel more focused, (C) both the same, (D) I haven't noticed any differences. Results showed that $63 \%$ of volunteers felt more focused after the Control, and $29 \%$ after the Verum situation (Figure 14).

\section{Discussion and Conclusion}

\subsection{Interpretation of the Results}

During the measurements, the situation for the volunteers was a bit stressful be- 
cause they had to perform the Clock test. Solving the test generates a tense psychological state. Therefore, by using the test we killed two birds with one stone: a) we were able to directly measure various attention parameters of volunteers and b) the situation was similar to the tense daily experience of many people. The applied MF stimulation was in this way closer to real-life than the majority of test situations that put volunteers under more artificial conditions. However, as described below, this situation brought also some drawbacks.

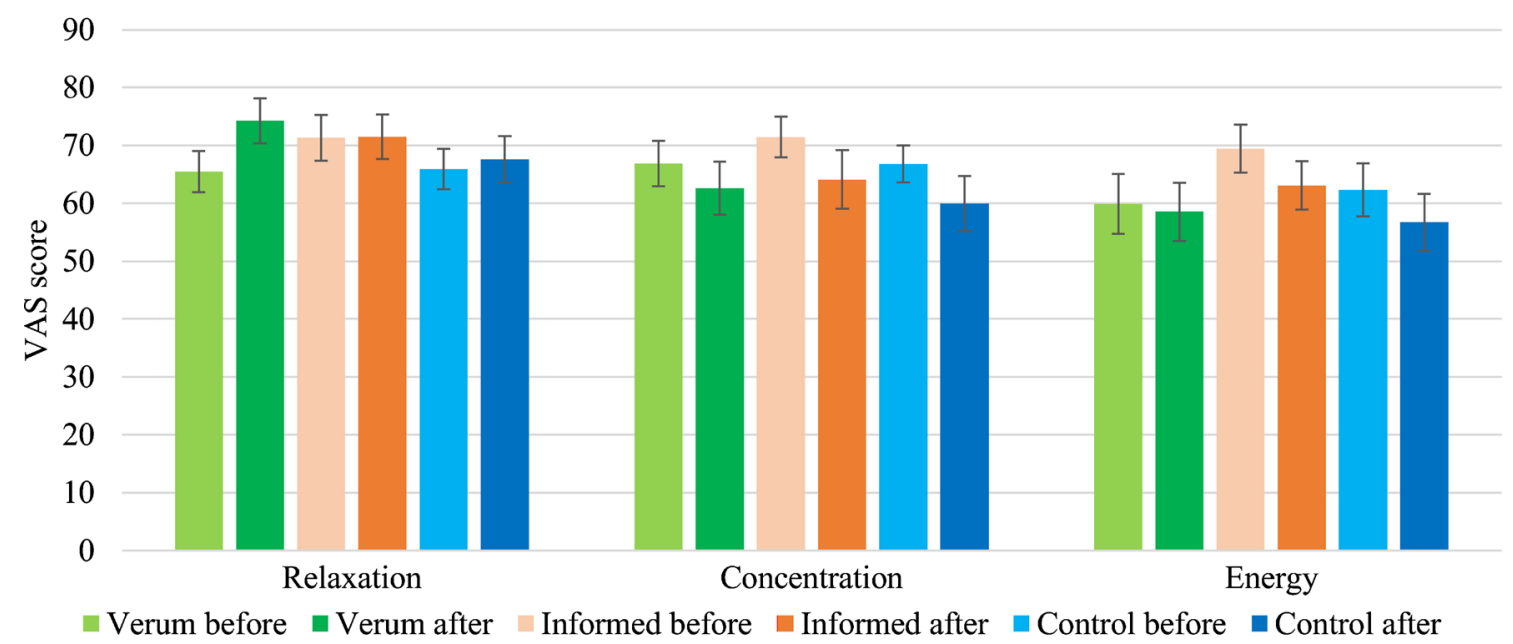

Figure 12. VAS scores of relaxation, concentration and energy level before and after the measurements (see the legend below graph bars) with Control, Verum and Informed. Shown are mean values \pm standard error $(\mathrm{N}=21)$.

Table 9. Comparison of changes in average VAS scores (values after-before) between Control and Verum situations $(\mathrm{N}=21)$.

\begin{tabular}{cc}
\hline VAS parameter & p value (paired t-test) \\
\hline Relaxation & 0.175 \\
Concentration & 0.666 \\
Energy & 0.566 \\
\hline
\end{tabular}

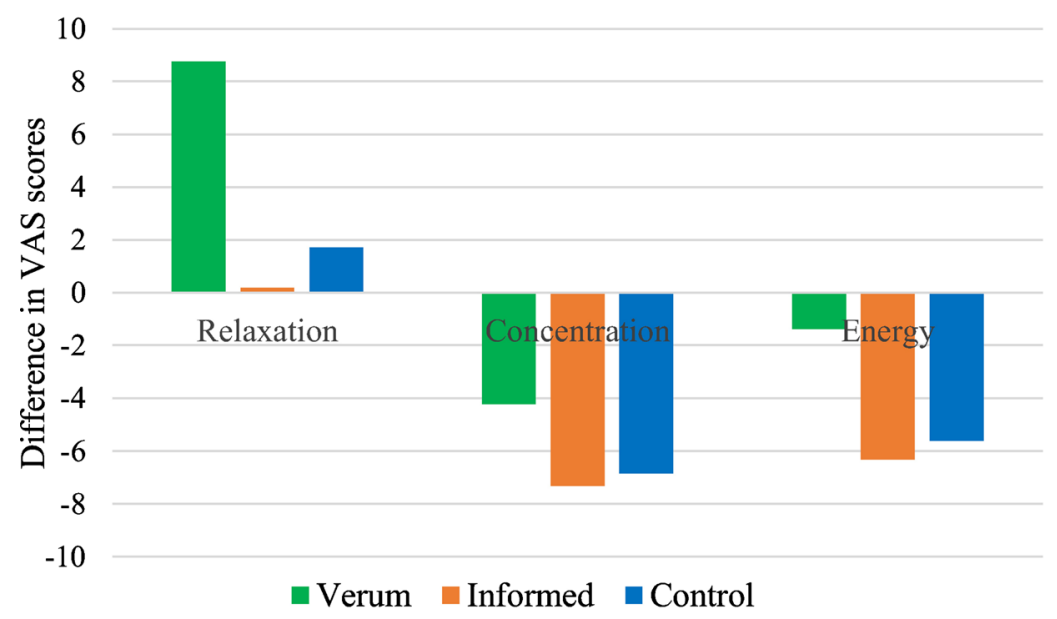

Figure 13. Differences in VAS score before/after the measurements for each parameter and all three situations that follow from the results presented in Figure 12. 


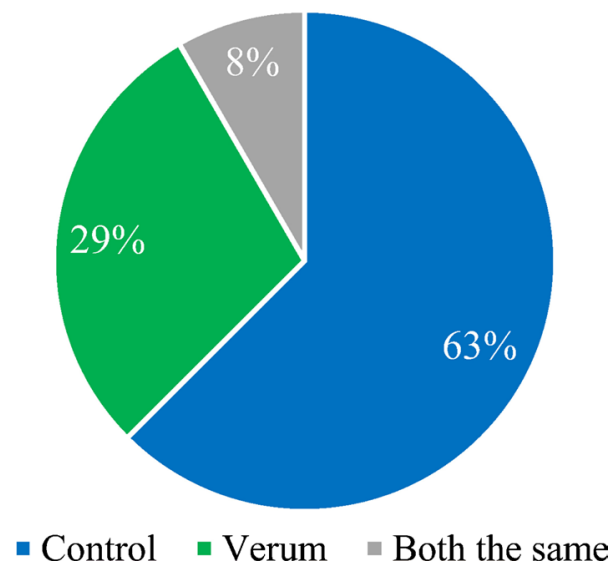

Figure 14. Volunteers' judgment of the situation that should provoke a higher focus after concluding both double-blind tests ( $\mathrm{N}=25$ volunteers).

In this respect, it is important and interesting that generally, the stimulation enhanced relaxation and worked in an anti-stress sense. The relaxing effect was strongly demonstrated by the SC parameter that can be seen even from the very beginning of measurements (see Figure 3) when the volunteers have already been stimulated for 10 minutes. Here, we may also observe some measure of an expectation (placebo-like, augmenting effect) at work, since the volunteers in Informed situation achieved significantly lower SC values than the ones in Verum. The difference even increases in the last 10 minutes of measurements. A similar outcome is given by HR (Figure 5), where the expectation seems to be at work more at the beginning while gradually fading towards the end of the measurement. The blind stimulation (Verum) effects, however, increased during measurements.

The parameters TED (Figure 4) and TMP (Figure 6) yielded statistically significant differences between Control and any of the MF stimulating situations (Verum or Informed). With both parameters, different as they are, the outcome speaks about a lower stress (see also [37] for TED and [38] for temperature). Especially with temperature, we may again see a positive influence of expectation that increased the effect of MF stimulation.

In general, the brainwaves' differences do not corroborate direct entrainment. This is most conspicuous with beta waves (Figure 9) that demonstrate no differences between situations. We assume the reason lies in the nature of the experimental situation involving the Clock test throughout measurements, which demanded a full commitment of the volunteer. Consequently, beta waves have already been at their high intensity and the entrainment could not show itself, which we regard as one of the above-mentioned drawbacks of this specific research situations. If we compare the proportions of beta waves in this experiment and the one involving relaxation entrainment research [39] with no task to be performed during the measurements, we observe that all beta values of the former are much higher than even the highest beta value of the latter (Figure $15)$. 


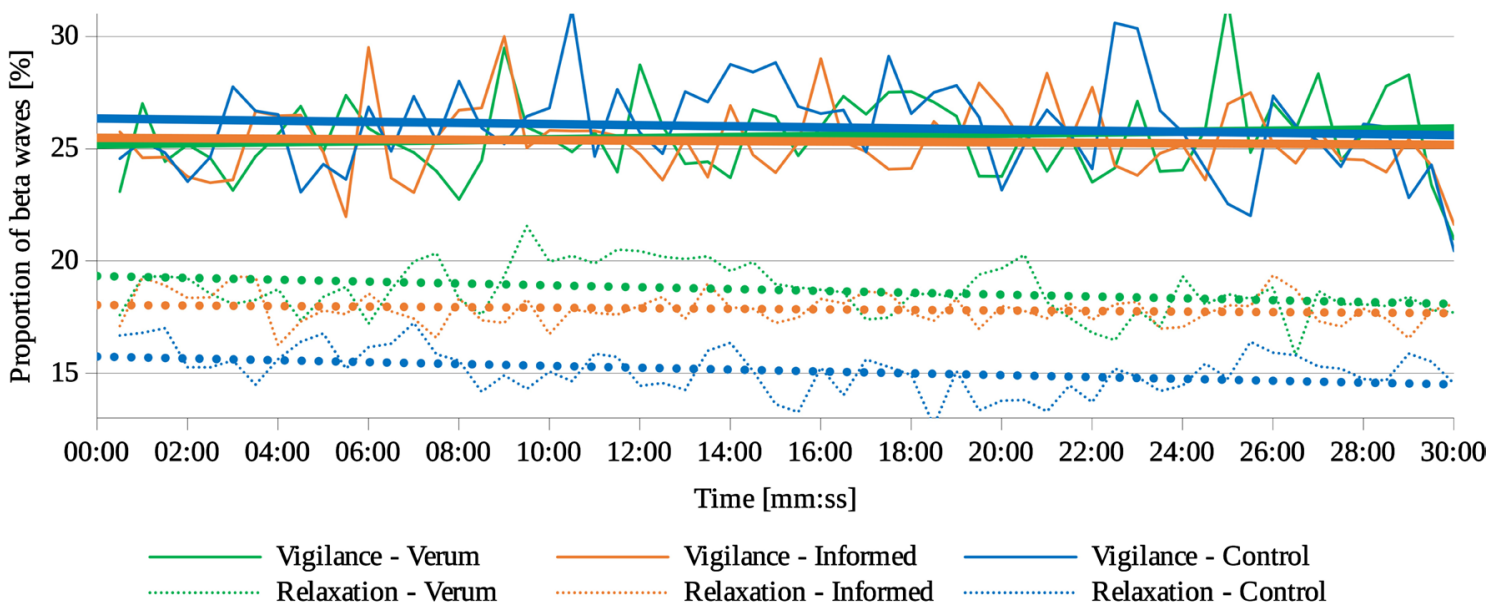

Figure 15. Beta values for three situations in the present vigilance research (solid lines) and the experiment with relaxation entrainment (dotted lines).

With alpha waves (Figure 7) we may observe an increasing trend in Verum, a weaker trend of the same kind in Informed and an opposite trend (decreasing) in Control. Here, the stimulated situations have the same sign again and are opposite to the blind non-stimulated one (Control). This further confirms the influence of the MF stimulation that may be a little modified by expectations and works in the direction of increasing relaxation. These brainwave measurement results highly conform to the results of other physiological tests measuring the autonomous nervous system's response.

Delta waves, however, demonstrate a different trend. In both MF stimulated situations their values are higher from the Control (significantly in case of Informed vs. Control) at the beginning (deeper relaxation), but become more or less equal to Control in the last measurement part. We interpret this as a gradual, although statistically not significant, effect that is in harmony with the entrainment model.

The VAS measurements, although not yielding significant differences, support the observed relaxing effect of MF stimulation on physiological parameters and alpha waves (Figure 13).

It is relevant to notice that in all cases of statistical differences between all three situations (see Table 4), the Informed situation differs from the Verum one in the same direction vs. Control, meaning that expectations of being stimulated in the direction of higher attention, work in the same sense-relaxing-as the MF stimulation itself. From this, we may indirectly infer that the applied MF stimulation supports vigilance. This is further corroborated by the Clock test results, which do not show a higher proportion of correctly detected skips or shifts (higher accuracy), yet demonstrate a) a significantly lower proportion of error responses of total dot shifts and b) a conspicuously lower mean reaction time with the Informed situation. Both these results speak in favor of higher attention, although the effect discloses itself in a stronger manner with the Informed situation where the conjunction between MF stimulation and expectations is at 
work.

\subsection{Conclusion}

- Statistically significant differences between the Verum and the Control situations (after Holm-Bonferroni corrections) for various physiological parameters strongly confirm the objective bioeffects of the stimulating MF of the Omnipemf device.

- The expectation of higher vigilance (present in Informed situation) improved the impact of the MF itself (Verum), which is mainly indicated by the same direction of the effect vs. Control, especially when there was a statistically significant difference between Verum and Informed (SC, TMP parameters). This was further confirmed in the Clock test with the error rate parameter and especially with the mean reaction time.

- However, we find the lowest number of significant differences regarding physiological parameters in comparisons between both stimulating situations, Verum and Informed, where only three such differences were found. This indicates that the applied MF itself induced the majority of differences against shame exposure and that expectations only improved this impact.

- Surprisingly, brain activity did not demonstrate important differences between different situations; consequently, the entrainment effect has not been confirmed. We assume that volunteers were already fully engaged in the Clock test so that their brain activity reached a plateau as we may convincingly perceive in Figure 15.

- VAS measurements in the relaxation parameter corroborated the electrophysiological ones.

- Relaxation, better nervous energy conservation and better performance (reduction of errors) may be concluded from all parts of the research.

- The fact that expectations of enhancing attention effect worked in the same sense as the blind MF stimulation corroborates the assumption that the MF stimulation regime used in the research acted in the direction of enhancing vigilance, although the volunteers, in general, did not recognize, when they were under stimulation (Figure 14).

\subsection{Guidelines for Future Research}

The future research on this line should include

- situations with no specific task to be performed during the measurements,

- duration of physiological and psychological changes after the end of stimulation,

- some other combinations of frequencies regarding optimization of entrainment,

- inclusion of positive control (guarana for instance) into the research,

- research of pure placebo (no stimulation, but told as if stimulated) influence,

- optimization of VAS questions,

- using some alternative tests measuring vigilance. 


\section{Acknowledgements}

This work was supported by MDCN Technologies Inc. New York, USA and MDCN Tech Ltd. Slovenia, EU. We would like to thank Vesna Periček Krapež and Mateja Senica for reviewing and correcting the manuscript.

\section{Conflicts of Interest}

The authors declare no conflicts of interest regarding the publication of this paper.

\section{References}

[1] Hoogendam, J.M., Ramakers, G.M. and Di Lazzaro, V. (2010) Physiology of Repetitive Transcranial Magnetic Stimulation of the Human Brain. Brain Stimulation, 3, 95-118. https://doi.org/10.1016/j.brs.2009.10.005

[2] Klomjai, W., Katz, R. and Lackmy-Vallée, A. (2015) Basic Principles of Transcranial Magnetic Stimulation (TMS) and Repetitive TMS (rTMS). Annals of Physical and Rehabilitation Medicine, 58, 208-213. https://doi.org/10.1016/j.rehab.2015.05.005

[3] Wassermann, E.M. (1998) Risk and Safety of Repetitive Transcranial Magnetic Stimulation: Report and Suggested Guidelines from the International Workshop on the Safety of Repetitive Transcranial Magnetic Stimulation, June 5-7, 1996. Electroencephalography and Clinical Neurophysiology, 108, 1-16. https://doi.org/10.1016/S0168-5597(97)00096-8

[4] Lednev, V.V. (1991) Possible Mechanism for the Influence of Weak Magnetic Fields on Biological Systems. Bioelectromagnetics, 12, 71-75. https://doi.org/10.1002/bem.2250120202

[5] Markov, M.S. (2007) Magnetic Field Therapy: A Review. Electromagnetic Biology and Medicine, 26, 1-23. https://doi.org/10.1080/15368370600925342

[6] Plankar, M., Brežan, S. and Jerman, I. (2013) The Principle of Coherence in Multi-Level Brain Information Processing. Progress in Biophysics and Molecular Biology, 111, 8-29. https://doi.org/10.1016/j.pbiomolbio.2012.08.006

[7] Meijer, D.K.F. and Geesink, J.H. (2019) Life and Consciousness Are Guided by a Semi-Harmonic EM Background Field. NeuroQuantology, 17, 37-44.

https://doi.org/10.14704/nq.2019.17.4.2074

[8] Baldwin, J. and Grantham, V. (2015) Radiation Hormesis: Historical and Current Perspectives. Journal of Nuclear Medicine Technology, 43, 242-246. https://doi.org/10.2967/jnmt.115.166074

[9] Roberts, B.M., Clarke, A., Addante, R.J. and Ranganath, C. (2018) Entrainment Enhances Theta Oscillations and Improves Episodic Memory. Cognitive Neuroscience, 9, 181-193. https://doi.org/10.1080/17588928.2018.1521386

[10] Bell, G.B., Marino, A.A. and Chesson, A.L. (1994) Frequency-Specific Responses in the Human Brain Caused by Electromagnetic Fields. Journal of the Neurological Sciences, 123, 26-32. https://doi.org/10.1016/0022-510X(94)90199-6

[11] Cvetković, D. and Čosić, I. (2009) Alterations of Human Electroencephalographic Activity Caused by Multiple Extremely Low Frequency Magnetic Field Exposures. Medical \& Biological Engineering \& Computing, 47, 1063-1073. https://doi.org/10.1007/s11517-009-0525-1

[12] Thut, G., Veniero, D., Romei, V., Miniussi, C., Schyns, P. and Gross, J. (2011) Rhythmic TMS Causes Local Entrainment of Natural Oscillatory Signatures. Cur- 
rent Biology, 21, 1176-1185. https://doi.org/10.1016/j.cub.2011.05.049

[13] Thut, G., Schyns, P. and Gross, J. (2011) Entrainment of Perceptually Relevant Brain Oscillations by Non-Invasive Rhythmic Stimulation of the Human Brain. Frontiers in Psychology, 2, 170. https://doi.org/10.3389/fpsyg.2011.00170

[14] Stodilka, R., et al. (2011) Pulsed Magnetic Field Exposure Induces Lasting Changes in Neural Network Dynamics. Neurocomputing, 74, 2164-2175. https://doi.org/10.1016/j.neucom.2011.01.025

[15] Dufor, T. (2017) Low Intensity rTMS to the Cerebellum: Age Dependent Effects and Mechanisms Underlying Neural Circuit Plasticity. Doctoral Dissertation, Paris, 6.

[16] Cook, C.M., Saucier, D.M., Thomas, A.W. and Prato, F.S. (2009) Changes in Human EEG Alpha Activity Following Exposure to Two Different Pulsed Magnetic Field Sequences. Bioelectromagnetics, 30, 9-20. https://doi.org/10.1002/bem.20434

[17] Cook, C.M., Thomas, A.W., Keenliside, L. and Prato, F.S. (2005) Resting EEG Effects during Exposure to a Pulsed ELF Magnetic Field. Bioelectromagnetics, 26, 367-376. https://doi.org/10.1002/bem.20113

[18] Ghione, S., Del Seppia, C., Mezzasalma, L. and Bonfiglio, L. (2005) Effects of $50 \mathrm{~Hz}$ Electromagnetic Fields on Electroencephalographic Alpha Activity, Dental Pain Threshold and Cardiovascular Parameters in Humans. Neuroscience Letters, 382, 112-117. https://doi.org/10.1016/j.neulet.2005.02.072

[19] Gao, X., Wang, X., Chen, F., Qi, H., Wang, X., Ming, D. and Zhou, P. (2014) Research on Brain Induced Effect by Extremely Low Frequency Pulsed Magnetic Stimulation. In: Engineering in Medicine and Biology Society (EMBC), 36th Annual International Conference of the IEEE, IEEE, Piscataway, 2613-2616.

[20] McIntire, L.K., McKinley, R.A., Goodyear, C. and Nelson, J. (2014) A Comparison of the Effects of Transcranial Direct Current Stimulation and Caffeine on Vigilance and Cognitive Performance during Extended Wakefulness. Brain Stimulation, 7, 499-507. https://doi.org/10.1016/j.brs.2014.04.008

[21] Mohri, Y., Kawaguchi, M., Kojima, S., Yamada, M., Nakano, T. and Mohri, K. (2017) Arousal Retention Effect of Magnetic Stimulation to Car Drivers Preventing Drowsy Driving without Sleep Rebound. Electronics and Communications in Japan, 100, 17-25. https://doi.org/10.1002/ecj.11949

[22] Casanova, M.F., Baruth, J.M., El-Baz, A., Tasman, A., Sears, L. and Sokhadze, E. (2012) Repetitive Transcanial Magnetic Stimulation (RTMS) Modulates Event-Related Potential (ERP) Indices of Attention in Autism. Translational Neuroscience, 3, 170-180. https://doi.org/10.2478/s13380-012-0022-0

[23] Sandyk, R. (1997) Immediate Recovery of Cognitive Functions and Resolution of Fatigue by Treatment with Weak Electromagnetic Fields in a Patient with Multiple Sclerosis. International Journal of Neuroscience, 90, 59-74. https://doi.org/10.3109/00207459709000626

[24] Lutz, A., Greischar, L.L., Rawlings, N.B., Ricard, M. and Davidson, R.J. (2004) Long-Term Meditators Self-Induce High-Amplitude Gamma Synchrony during Mental Practice. Proceedings of the National Academy of Sciences, 101, 16369-16373. https://doi.org/10.1073/pnas.0407401101

[25] Rubik, B. (2011) Neurofeedback-Enhanced Gamma Brainwaves from the Prefrontal Cortical Region of Meditators and Non-Meditators and Associated Subjective Experiences. The Journal of Alternative and Complementary Medicine, 17, 109-115. https://doi.org/10.1089/acm.2009.0191

[26] Geesink, J.H. and Meijer, D.K.F. (2017) Electromagnetic Frequency Patterns That Are Crucial for Health and Disease Reveal a Generalized Biophysical Principle: The 
GM Scale. Quantum Biosystems, 8, 1-16.

[27] Henry, M. (2016) Hofmeister Series: The Quantum Mechanical Viewpoint. Current Opinion in Colloid \& Interface Science, 23, 119-125.

https://doi.org/10.1016/j.cocis.2016.08.001

[28] INTERMAGNET (2019) International Real-Time Magnetic Observatory Network. http://www.intermagnet.org/data-donnee/dataplot-eng.php?type=hdz

[29] Mueller, S.T. (2011) PEBL's Clock Test. Computer Software. http://pebl.sf.net/battery.html

[30] Mackworth, N.H. (1948) The Breakdown of Vigilance during Prolonged Visual Search. Quarterly Journal of Experimental Psychology, 1, 6-21. https://doi.org/10.1080/17470214808416738

[31] Jasper, H.H. (1958) The Ten-Twenty Electrode System of the International Federation. Electroencephalography and Clinical Neurophysiology, 10, 370-375.

[32] Holm, S. (1979) A Simple Sequentially Rejective Multiple Test Procedure. Scandinavian Journal of Statistics, 6, 65-70.

[33] Heller, G.Z., Manuguerra, M. and Chow, R. (2016) How to Analyze the Visual Analogue Scale: Myths, Truths and Clinical Relevance. Scandinavian Journal of Pain, 13, 67-75. https://doi.org/10.1016/j.sjpain.2016.06.012

[34] Wilhelmus, M.M., et al. (2017) Effects of a Single, Oral $60 \mathrm{mg}$ Caffeine Dose on Attention in Healthy Adult Subjects. Journal of Psychopharmacology, 31, 222-232. https://doi.org/10.1177/0269881116668593

[35] 1KA (2019) 1KA (Version 19.06.07) (Software). Faculty of Social Sciences, Ljubljana. https://www.1ka.si

[36] Borchardt, A.R. and Zoccola, P.M. (2018) Recovery from Stress: An Experimental Examination of Focused Attention Meditation in Novices. Journal of Behavioral Medicine, 41, 836-849. https://doi.org/10.1007/s10865-018-9932-9

[37] Nice, L.B., Rock, J.L. and Courtright, R.O. (1914) The Influence of Adrenaline on Respiration. American Journal of Physiology-Legacy Content, 34, 326-331. https://doi.org/10.1152/ajplegacy.1914.34.3.326

[38] Vinkers, C.H., Penning, R., Hellhammer, J., Verster, J.C., Klaessens, J.H., Olivier, B. and Kalkman, C.J. (2013) The Effect of Stress on Core and Peripheral Body Temperature in Humans. Stress, 16, 520-530. https://doi.org/10.3109/10253890.2013.807243

[39] Jerman, I., Dovč, P. and Ratajc, P. (2019) Influencing Relaxation by a Low Intensity Transcranial Pulsed Magnetic Stimulation Applying the Entrainment Model. OALib Journal, 6, 5741. https://doi.org/10.4236/oalib.1105741 